\title{
Distribution, classification, domain architectures and evolution of prolyl oligopeptidases in prokaryotic lineages
}

\author{
Swati Kaushik ${ }^{1,2}$ and Ramanathan Sowdhamini $1^{{ }^{*}}$
}

\begin{abstract}
Background: Prolyl oligopeptidases (POPs) are proteolytic enzymes, widely distributed in all the kingdoms of life. Bacterial POPs are pharmaceutically important enzymes, yet their functional and evolutionary details are not fully explored. Therefore, current analysis is aimed at understanding the distribution, domain architecture, probable biological functions and gene family expansion of POPs in bacterial and archaeal lineages.

Results: Exhaustive sequence analysis of 1,202 bacterial and 91 archaeal genomes revealed 3,000 POP homologs, with only 638 annotated POPs. We observed wide distribution of POPs in all the analysed bacterial lineages. Phylogenetic analysis and co-clustering of POPs of different phyla suggested their common functions in all the prokaryotic species. Further, on the basis of unique sequence motifs we could classify bacterial POPs into eight subtypes. Analysis of coexisting domains in POPs highlighted their involvement in protein-protein interactions and cellular signaling. We proposed significant extension of this gene family by characterizing 39 new POPs and 158 new a/ $\beta$ hydrolase members.
\end{abstract}

Conclusions: Our study reflects diversity and functional importance of POPs in bacterial species. Many genomes with multiple POPs were identified with high sequence variations and different cellular localizations. Such anomalous distribution of POP genes in different bacterial genomes shows differential expansion of POP gene family primarily by multiple horizontal gene transfer events.

Keywords: Prolyl oligopeptidase, Genome wide survey, Phylogeny, Bacterial prolyl oligopeptidase

\section{Background}

Proteases are degradation machines that aid in the proper functioning of cells by sustaining the balance between protein synthesis and hydrolysis. The proteolytic enzymes are also involved in post-translational modifications and generation of active peptide molecules in the cell [1]. In fact, almost $2 \%$ of all proteins in the cell are proteolytic in nature, highlighting the decisive role of proteolytic enzymes in cellular regulatory circuits $[2,3]$. Serine proteases are family of proteases that can cleave a peptide bond using a nucleophilic serine residue in their catalytic triad. They are involved in diverse biological processes, and are considered as attractive targets for drug design $[4,5]$. Prokaryotic serine proteases play an

\footnotetext{
* Correspondence: mini@ncbs.res.in

${ }^{1}$ National Centre for Biological Sciences, Tata Institute of Fundamental Research, GKVK Campus, Bellary Road, Bangalore 560065, India

Full list of author information is available at the end of the article
}

important role in cell signaling, metabolism and various defense responses [1,6,7], and help these microorganisms to adapt to a wide variety of environments and growth conditions $[8,9]$.

A particular type of serine protease that can hydrolyse internal proline residues distinctly is referred as prolyl oligopeptidase (POP, family S9A, according to MEROPS database) $[2,10,11]$. POPs are distinct from other proteases that cannot cleave peptide bonds formed by proline residues due to its imino ring structure. POPs are highly selective as their oligopeptidase activity is restricted to the substrates of up to 30 amino acids [12]. This specificity in cleaving short peptides and exclusion of large proteins make them unique in nature. POPs are widely distributed in all the domains of life ranging from bacterial and archaeal species to humans [13]. However, unlike other serine proteases, the exact physiological role, 
genomic distribution and evolutionary details of POPs in bacteria is still unknown.

In most of the species, POPs are $\sim 700$ residues long with a cylindrical structure [14]. Crystal structure of bacterial POPs (bPOPs) from Myxococcus xanthus, Sphingomonas capsulata and Aeromonus punctata suggested two domain architecture with a sequentially discontinuous catalytic $\alpha / \beta$ hydrolase and a $\beta$-propeller domain $[15,16]$. The $\alpha / \beta$ hydrolase domain in POPs consists of a short helical ( $\sim 70$ residue) N-terminal stretch and a large C-terminal region comprising of catalytic triad. The catalytic triad of Ser, Asp and His is hidden at the interface of the two structural domains. Recently, seven crystal structures of POPs of Aeromonus punctata suggested induced-fit mechanism of substrate entry, where addition of a substrate induces large-scale conformational changes in two domains along with alterations at the active site [16]. Studies have shown the ability of the bPOPs to cleave even 33-mer peptides [17]. POPs from different bacteria can also have differences in chain-length and sub-site specificity towards substrates [17].

POPs are one of the members of the larger 'POP family' (S9 in MEROPS), which also includes dipeptidyl peptidase IV (DPP, S9B), acylaminoacyl peptidase (ACC, S9C) and oligopeptidase B (OPB, S9A) [2,11]. All the members of POP family are ubiquitous and exhibit restricted substrate specificities. While POP hydrolyses peptides at the carboxyl side of proline residue [12], DPP liberates dipeptides where penultimate amino acid is proline [18]. OPB cleaves at arginine and lysine bonds [19] and ACC remove $\mathrm{N}$-acetylated amino acids from blocked peptides [20]. DPPs are homodimers and exist in both soluble and membrane bound forms [21-23]. POP and OPB are cytoplasmic endopeptidases, while DPP and ACC are exopeptidases. Though the sequence similarity of these four peptidases is low, they have similar three-dimensional structures with catalytic hydrolase and propeller domains. The propeller domain of POP, ACC and OPB is seven bladed, as compared to more irregular eight bladed propeller of DPP $[24,25]$.

POPs and POP family members are pharmaceutically important enzymes. bPOPs are considered as therapeutic agents for the oral treatment of celiac sprue, which is a small-intestinal disorder caused by abnormal response to gluten proteins [26]. High proline content of gluten makes it resistant to digestion by enzymes present in gastrointestinal tract. Treatment of gluten peptides with POPs from Flavobacterium meningosepticum, Sphingomonas capsulatum and Myxococcus xanthus has shown rapid cleavage of them [18]. Physiological role of the prokaryotic DPPs is not very clear, but there is evidence suggesting their involvement in virulence of Porphyromonas gingivalis, which is a major pathogen associated with adult periodontitis [27]. Similarly, OPBs are involved in the pathogenicity of $T$. brucei, the causative agent of African trypanosomiasis [9]. In trypanosome both POPs and OPBs are considered to be important virulence factor [28].

The availability of genomic information of many bacterial and archaeal species offers a great opportunity to understand the detailed distribution and biochemical role of POPs in prokaryotic lineages. Motivated by the clinical importance of POPs, we have carried out genomic identification of POPs and its homologs using exhaustive sequence search procedures. We found POPs to be widely distributed in all the classes of bacteria and archaea with diverse domain architectures. These bPOPs were depicted to be involved in protein-protein interactions and cellular signaling. Rigorous sequence searches employed in this study aided the identification of many additional POPs, which were not characterized earlier. Detailed clustering and identification of class specific sequence motifs allowed classification of bPOPs into eight subtypes. We found that multiple horizontal gene transfer events were responsible for the differential expansion of POP gene family in bacteria. To our knowledge, this is the first analysis that reports the presence of multiple POPs in many bacterial genomes.

\section{Results and discussion}

Genomic identification of POP homologs in bacteria and archaea

We first implemented direct and profile-based sensitive sequence search methods to identify POP homologs from 23 bacterial and 4 archaeal phyla (Additional file 1S1a and Additional file 2). Hits were considered as 'true', if the sequence search algorithms could identify them with both $\beta$-propeller (POP $N$ ) and $\alpha / \beta$ hydrolase (POP_C) domains, or with at least $\alpha / \beta$ hydrolase domain. At a stringent E-value of $10^{-10}$, only 1,791 POP homologs could be identified, while relaxing the E-value to $10^{-3}$ could capture 3,387 additional POP homologs (Additional files 3 and 4). In total, 3,010 POP homologs were collected using exhaustive Phmmer, Jackhmmer and profile-based approaches, including 2,919 bacterial and 91 archaeal POP homologs $[29,30]$.

The collected hits included annotated POPs, POP family members and nearby hydrolases of $\alpha / \beta$ hydrolase superfamily. Altogether, they are referred as 'POP homologs' in this report. In certain bacterial (Aquificae, Deferribacteres, Elusimicrobium, Dictyoglomi, Tenricutes) and archaeal (Nanoarchaeota and Thaumarchaeota) lineages no POP homologs could be identified. BLAST searches also failed to capture POP homologs in these phyla except in Dictyoglomi [31]. However, sequence searches against appended-PALI + database could pick at least one POP homologue in the above phyla except for Nanoarchaeota [32]. 


\section{Wide distribution of POP homologs in prokaryotic lineages}

We noticed that all the collected POP homologs were widely distributed across all the major lineages of bacteria and archaea with apparent loss in Nanoarchaeota. Phylum Actinobacteria was identified to be the most populated with 1000 POP homologs (Figure 1), while in archaea, many POP homologs were captured from Euryarchaeota and Crenarchaeota. In POP family, POPs were more abundant $(44 \%)$ in prokaryotic lineages than DPPs (24\%) and OPBs (10\%) (Figure 1c Additional file 5). We could also capture all the 638 annotated POPs from prokaryotes.

\section{Bacterial POP homologs are both secretory and membrane proteins}

Earlier studies have shown that bPOPs are associated with the signal peptides [13]. Signal peptides are sequence motifs that permit the proteins to translocate across endoplasmic reticulum in eukaryotes and to the cytoplasmic membrane in prokaryotes. Therefore, we examined all the collected POP homologs for the presence of signal peptides. Our results showed that $20 \%$ of the POP homologs were predicted to be associated with such signals, from which 225 (35\%) were annotated POPs (Figure 2). Bacteroides (78\%) and Acidobacteria (75\%) had maximum number of POP homologs with signal peptides, while in some bacterial phyla (e.g. Fusobacteria, Spirochaetes, Thermotogae and Synergistes) signal peptides were completely absent. POP homologs from gram-positive bacterial phyla (Actinobacteria and Firmicutes) showed relatively less number of signal peptides.

Recently, membrane-bound forms of POPs isolated from synaptosomal membranes of bovine brain were also reported [33,34]. Cytosolic and membrane forms are different with respect to sensitivity to inhibitors, relative molecular mass, affinities for the peptide substrate and the presence of a hydrophobic membrane anchor [33,34]. Transmembrane helix prediction by TMHMM identified 236 annotated bPOPs with single transmembrane helices located at the N-terminal [35]. Transmembrane helices were absent in POPs of phyla Spirochaetes and Fusobacteria.

\section{Diverse domain architectures reveal putative functions of POP homologs}

We then investigated the coexisting domains to understand the possible biological functions of POP homologs in the prokaryotic lineages. Bacterial and archaeal POP homologs were associated with 105 and 8 different domain architectures respectively (Figure 3, Additional file 6). Both the archaeal and bacterial POP homologs share similar domain architectures suggesting similar function of POP homologs in these two kingdoms. Domain architectures of POP homologs were also mapped on species tree of bacteria and archaea. As shown in Figure 4, POPs were associated with diverse domain combinations in Proteobacteria, while in mycobacterial species POPs were replaced by other hydrolases. Within a phylum, anomalous distribution of POPs was observed. Mapping of domain architecture on archaeal species tree depicted presence of only C-terminal POP domain in most of the organisms, while full-length POP domains were observed in a few species of Crenarchaeota (Figure 5).

POP homologs were frequently associated with proteinprotein interaction domains e.g. PDZ and tetratricopeptide (TPR) repeats. Two of the 'C-terminal processing peptidases' (S41) had PDZ domains, which are associated with

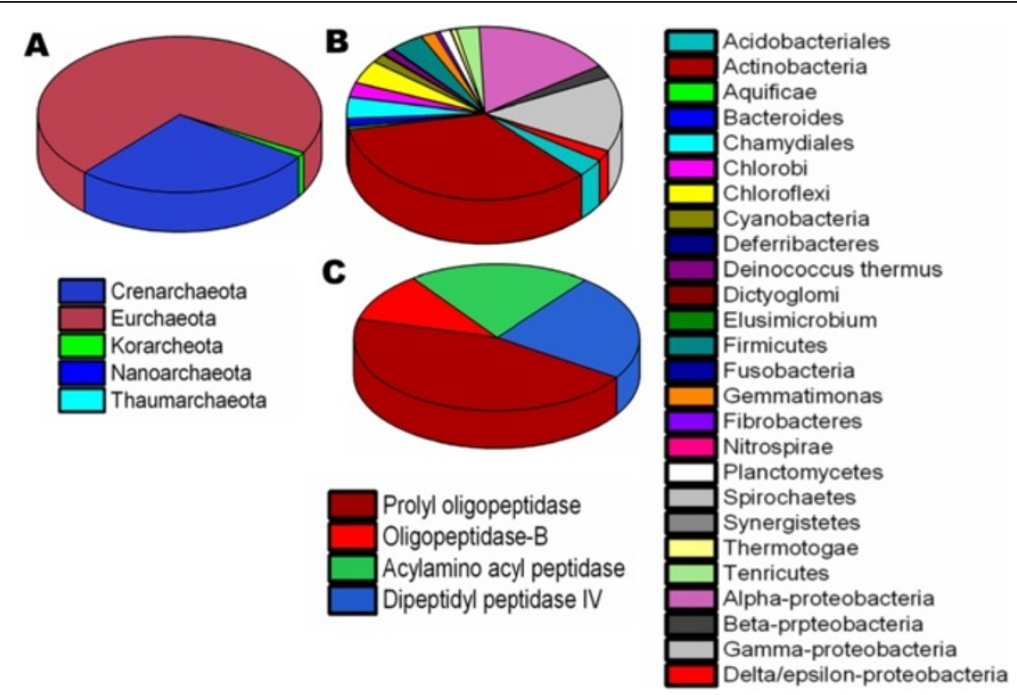

Figure 1 Distribution of POP homologs in prokaryotic lineages. A) Distribution of archaeal POP homologs. B) Distribution of bacterial POP homologs. C) Distribution of POP family members. 


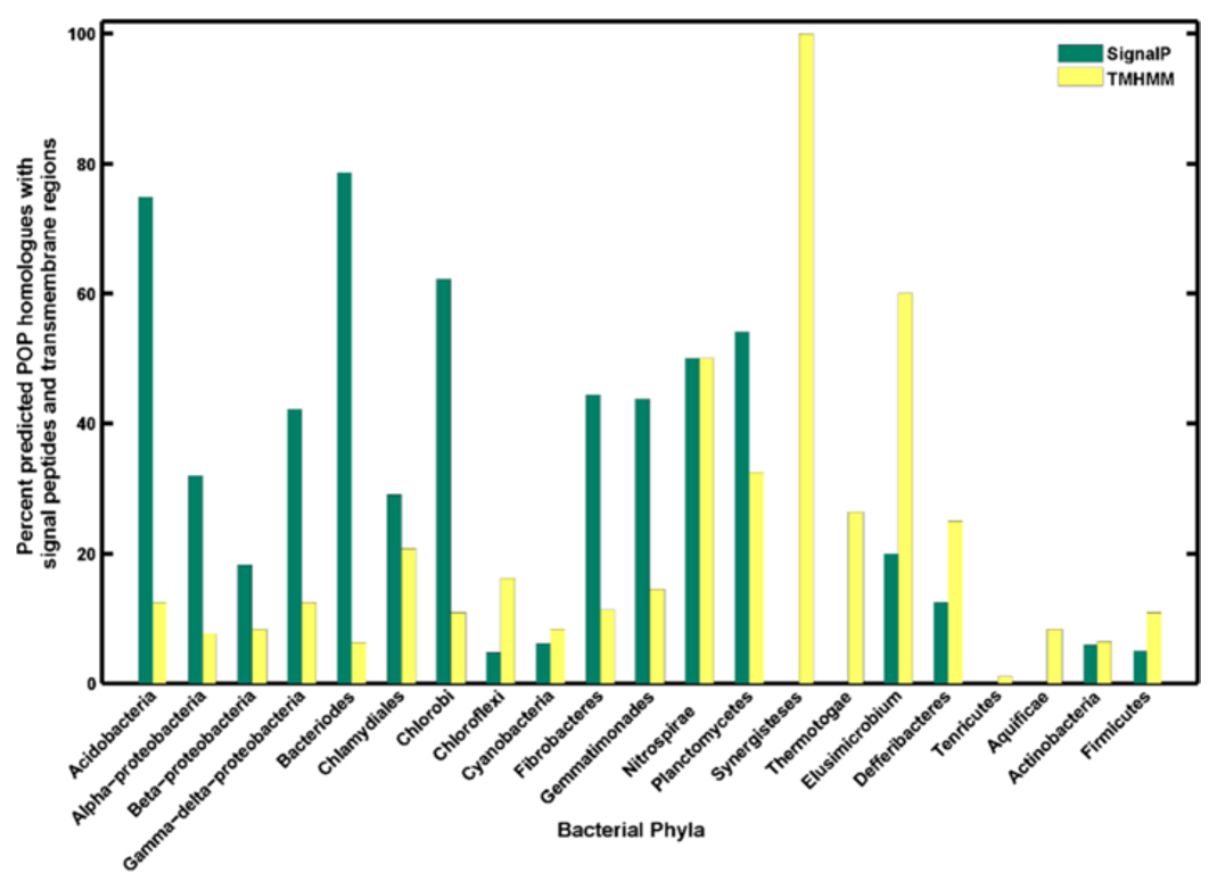

Figure 2 Bacterial POP homologs with signal peptides and transmembrane regions.

signaling proteins of bacteria, plants and higher order organisms. PDZ domains are involved in assembly of large protein complexes, thereby coordinating and guiding the flow of regulatory information [36,37]. PDZ domains present in peptidase S41 of Candidatus Solibacter usitatus (YP_821861) and Roseiflexus (YP_001276641) were associated with WD40 and DPP domains. TPR repeat motifs facilitate interaction with other proteins. These motifs were also related with hydrolase domain in Candidatus Solibacter usitatus (YP_824720). TPR-proteins are also associated with multi-protein complexes, and are involved in functioning of chaperones, cell-cycle, transcription and protein transport complexes [38,39].

POP homologs were also associated with signaling modules such as WD-repeats. Proteins with WD-repeat exhibit high degree of functional diversity [40-42]. Some archaeal POPs were also predicted to be associated with WD-repeats suggesting conserved function of POPs in the two domains of life. Besides WD repeats, POP homologs were also related with Sel1 repeats, which are subfamily of TPR sequences. In prokaryotes, these repeats allow proteins to be membrane attached and mediate interaction between bacterial and eukaryotic host cells $[43,44]$. One of the POP proteins from Ferrimonas balearica (YP_003914375) was predicted to be associated with Sel1 repeats.

Bacterial POP homologs were also found to co-exist with several DNA-binding modules of transcription regulatory domains. Numerous bacterial transcription regulatory proteins bind DNA via a helix-turn-helix motif [45]. These are sequentially diverse transcriptional activators and most of them are known to negatively regulate their own expression. Transcription regulatory domain is associated with response regulator receiver domain and plays an important role in DNA-binding and regulation of transcription [46,47]. POP homologs that co-existed with bacterial regulatory domains include Candidatus Solibacter usitatus (YP_827731) and Caulobacter segnis (YP_003594106), and those with transcription regulatory domain include four homologs (two each from Actinobacteria and Gammaproteobacteria (YP_888147 (Mycobacterium smegmatis), YP_001759306 (Shewanella woodyi), YP_735011 (Shewanella sp. MR-4) and YP_954217 (Mycobacterium vanbaalenii)). Targeted deletions of the predicted accessory domains will be beneficial to understand the related biological functions.

\section{Different cellular localization of annotated bPOPs}

We have also examined the cellular localization of annotated bPOPs to infer the possible functions of POP in more detail. Prediction of cellular localization using PSORT-b also revealed cytoplasmic nature of the annotated POPs (176 versus 115 POPs which were predicted to be periplasmic) (Additional files 4 and 7) [48]. Interestingly, we predicted some of these POPs to be localized in cell wall, cytoplasm and outer membranes of bacteria and archaea. Different bacterial phyla depicted differences in preferred cellular localization of POPs. For example, in phylum Proteobacteria, most of the POPs were periplasmic in nature. Clustering analysis of the 


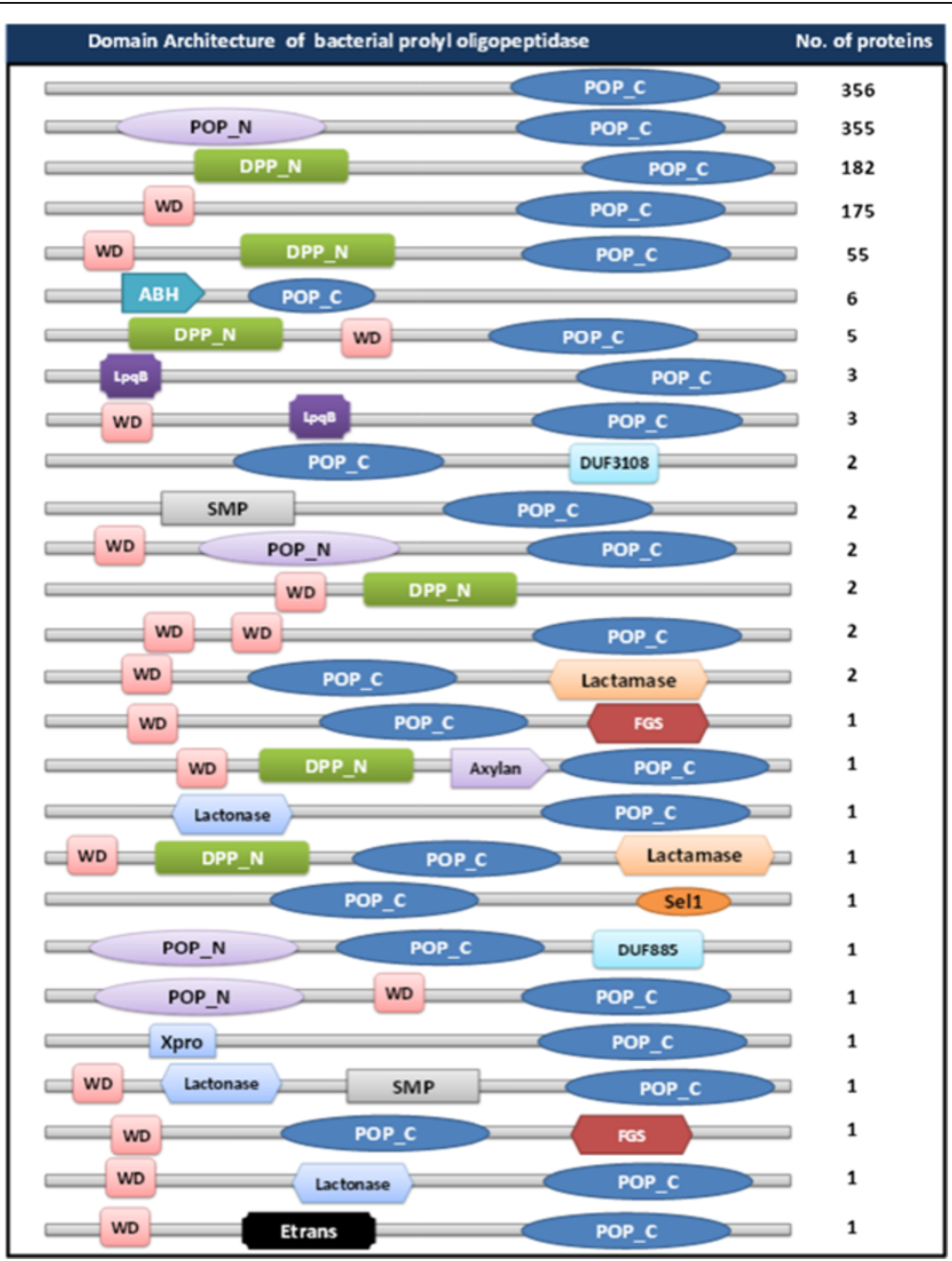

Figure 3 Domain architecture of annotated bPOPs. Abbreviations: POP_N-prolyl oligopeptidase N-terminal, POP_C-prolyl oligopeptidase C-terminal, DPP_N-Dipeptidyl peptidase N-terminal, WD-WD domain, ABH-a/ß hydrolase, LpqB -Lipoprotein,DUF- Domain of unidentified function, SMP-SMP-30/gluconolactonase/LRE-like region, FGS-Formylglycine-generating sulfatase, Axylan-Acetyl xylan, Xpro -X-Pro dipeptidyl-peptidase, Etrans-Eukaryotic translation.

predicted cytoplasmic and periplasmic POPs resulted in a clear separation of cytoplasmic and periplasmic POPs with a few exceptions.

\section{Phylogenetic analysis of annotated bPOPs shows high co-clustering}

To investigate the differences in the annotated bPOPs, we next performed phylogenetic clustering of 638 annotated POPs that showed nine distinct clusters, with coclustering among members of different phyla (Figure 6). This co-clustering trend and absence of phylum-specific clusters suggested high conservation of POPs within bacterial lineages. Genus Shewanella of marine metalreducing bacteria was highly populated with considerable number of annotated bPOPs in all the nine clusters. Similarly, archaeal POPs were also co-clustered well with other bPOPs. This co-clustering suggested the possibility of lateral transfer of POP genes among bacteria and between archaeal and bacterial species (Additional files 4 and 8).

\section{Unique sequence signature motifs depict diverse sequence properties}

To further analyse the co-clustering trend of annotated bPOPs, we identified conserved class specific sequence motifs. An alignment stretch was considered as a 'conserved motif', if $95 \%$ of the sequences had conserved amino acids at least at three consecutive positions. From these highly conserved sequence motifs, we next identified class specific motifs. A 'class-specific motif' was defined as a sequence motif in a cluster, which was completely absent from all the other clusters. In the first and seventh clusters (Figure 6), no class-specific motifs were observed. Figure 7 shows a part of the alignment of fifth 


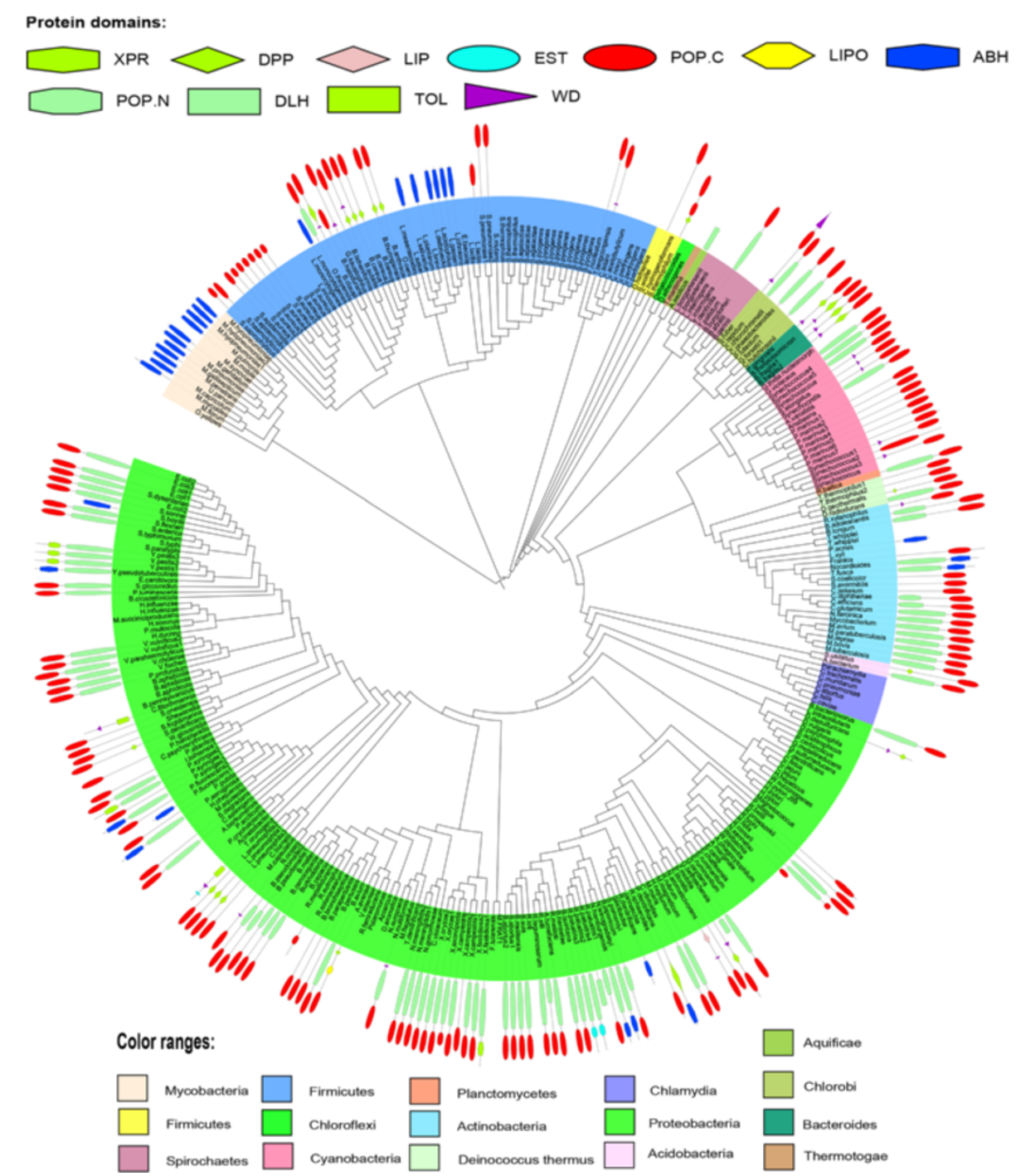

Figure 4 Domain architecture of POP homologs mapped on the species tree of bacteria. Abbreviations: DPP-Dipeptidyl peptidase, LIP-Lipoprotein LpqB, EST-esterase, POP_N-prolyl oligopeptidase N-terminal domain, POP_C-prolyl oligopeptidase C-terminal domain, LIPO-lysophospholipase, ABH-a/ $\beta$ hydrolase, XPR-X-Pro dipeptidyl-peptidase, DLH-Dienelactone hydrolase, TOL-TolB amino-terminal, WD-WD domain.

cluster of bPOPs representing class specific motifs. Detailed analysis of motifs of all the clusters was carried out to understand their relative position on the structure of bPOPs. Class-specific motifs of second, sixth, eighth and ninth cluster were localized in the hydrolase domain, while motifs of cluster third, fourth and fifth were distributed on both the domains (see Additional files 9 and 10 for details).

\section{Classification of annotated bPOPs into eight subtypes}

Detailed analysis of class specific sequence motifs indicated high sequence variations in annotated bPOPs. Therefore, on the basis of identified class specific motifs, we propose a classification of bPOPs into eight different subtypes as shown in Figure 8. Some of these classspecific motifs were surface exposed, depicting their possible involvement in protein-protein interactions with other interacting partners (for details see Additional file 4), while some other motifs were located in the core of protein, near functionally important residues, which could possibly cause differences in interaction with the versatile substrates of POPs.

\section{Subtypes of bPOPs differ in the conservation of functionally important residues}

We then investigated the conservation of functionally important residues in different subtypes of bPOPs. Detailed analysis revealed high conservation of catalytic triad residues in all the subtypes. However, high number of non-permissible amino acid replacements was observed to be concentrated at two sites-Ser-571 and Thr-573 


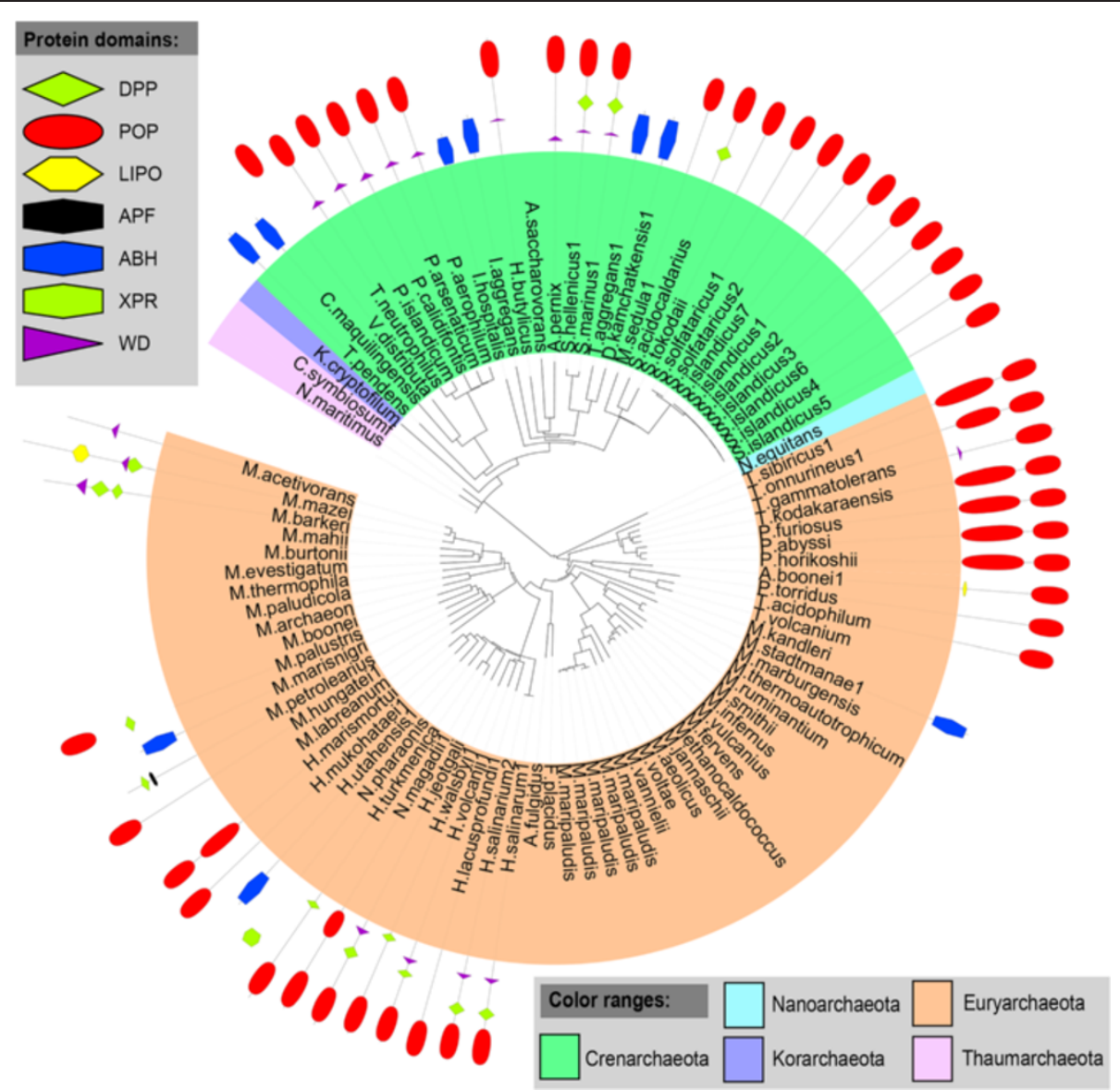

Figure 5 Domain architecture of POP homologs mapped on the species tree of archaea. Abbreviations: DPP-Dipeptidyl peptidase, POP_N-prolyl oligopeptidase N-terminal domain, POP_C-prolyl oligopeptidase C-terminal domain, LIPO-lysophospholipase, APF-Anaphase-promoting complex, ABH-a/ $\beta$ hydrolase, XPR-X-Prodipeptidyl-peptidase, WD-WD domain.

(numbering according to the bPOP crystal structure, PDB id: $2 \mathrm{BKL}$ ), which are located at the interface of two domains. These sites were replaced by non-polar and positively charged amino acids in most of the subtypes of bPOPs (Additional file 11). These two residues were also situated in vicinity of Arg-572 and Ile-575 that were reported to be crucial for the incoming peptide substrate in bPOPs. W-575, which is important for the substrate binding was conserved in some of the bPOPs, while in a few other bPOPs it was substituted by other amino acids. Altogether, the hydrophobic environment required for the substrate binding was not conserved in all the bPOPs. These findings strengthen our hypothesis that the proposed bPOP subtypes can also be different with respect to the possible substrate. Mutation experiments of these functionally important residues can provide further insights about their role in the catalytic activity and substrate specificity.

\section{Divergence of POP family members}

Besides analysing the co-clustering pattern of annotated bPOPs, we have also examined the divergence of POP family members. From all the 3,010 collected POP homologs, we could obtain 1,421 POP family members including 638 annotated POPs, 156 OPBs, 293 ACCs and 334 DPPs. These members were used to construct a joint phylogenetic tree, where a set of bacterial carboxylesterases (20 sequences) were considered as an outgroup. We observed that OPBs and DPPs were distinctly clustered, while the ACCs and POPs were dispersed all over the tree (Additional files 12 and 13). bPOP family tree was contradictory to the tree earlier reported by Venäläinen et al., where distinct clusters of POP family members from all the domains of life were observed [13].

The phylogenetic analysis also suggested high divergence of other POP family members. Some of the POPs have diverged from the rest of the POP family members before OPBs, followed by the divergence of ACCs and DPPs. ACCs were diverged along with some of the other POPs, since distinct cluster of ACCs could not be obtained. This clustering pattern was confirmed by generating additional phylogenetic tree, where only DPPs, OPBs and ACCs were considered to understand their phyletic 


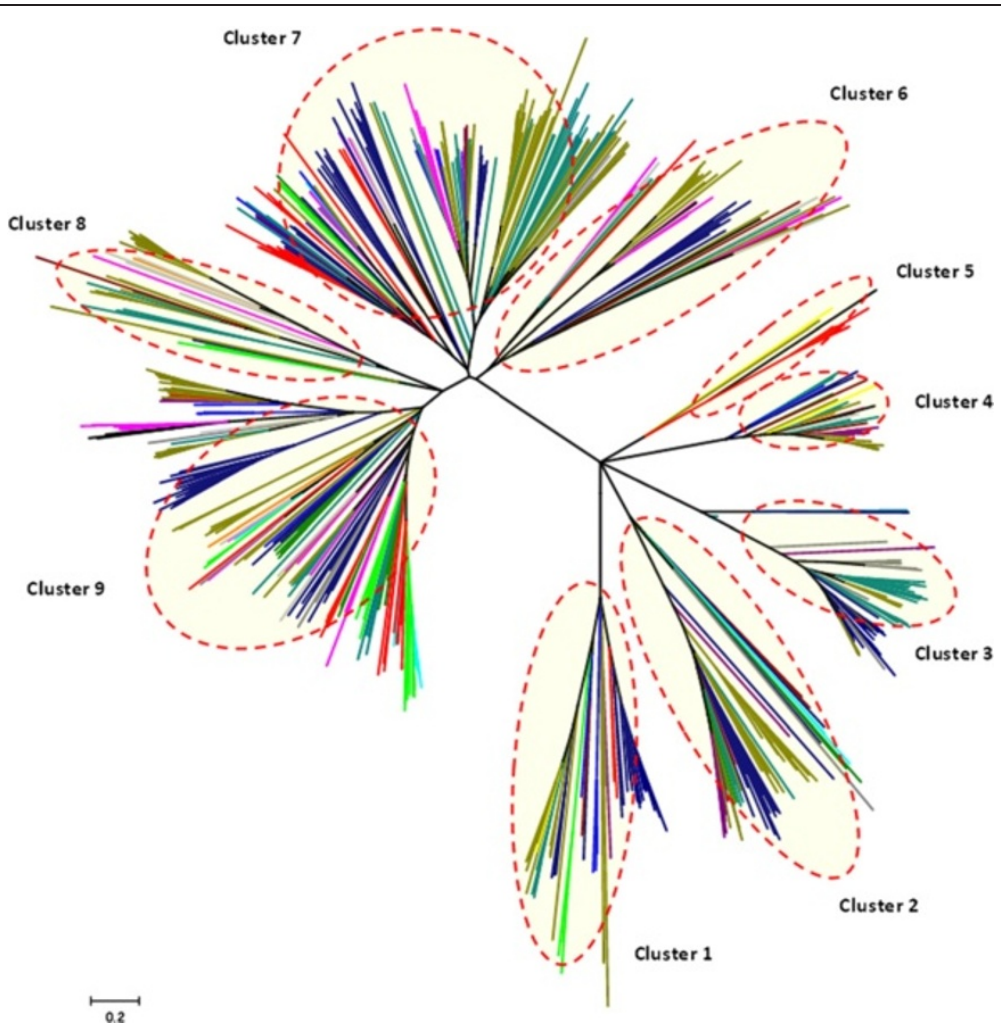

Figure 6 Phylogenetic analysis of annotated bPOPs. Color code: Thermotogae-cyan, Firmicutes-lime, Chloroflexi-green, Deinococcus-thermus-blue, Chlorobi-magenta, Actinobacteria-blue, Acidobacteria-yellow, Alphaproteobacteria-teal, Betaproteobacteria-grey, Gammaproteobacteria-olive, Deltaproteobacteria-blue, Bacteriodetes-black, Planctomycetes-black, Cyanobacteria-purple, Gemmatimonadetes-Red branch with species name in black, Spirochaetes-pink branch with species name in black, Fibrobacteres-light grey, Archaebacteria-red. Nine distinct clusters are marked in red.

distribution (Additional file 4). If POPs were excluded from the phylogenetic tree, other members of POP family formed distinct clusters, which revealed that POPs were responsible for the observed co-clustering among the POP family members.
Anomalous distribution of annotated bPOPs revealed many multi-POP bacterial genomes

While performing the sequence analysis, we noticed high variations in the number of annotated POP genes in bacterial genomes, ranging from no POPs to multiple copies

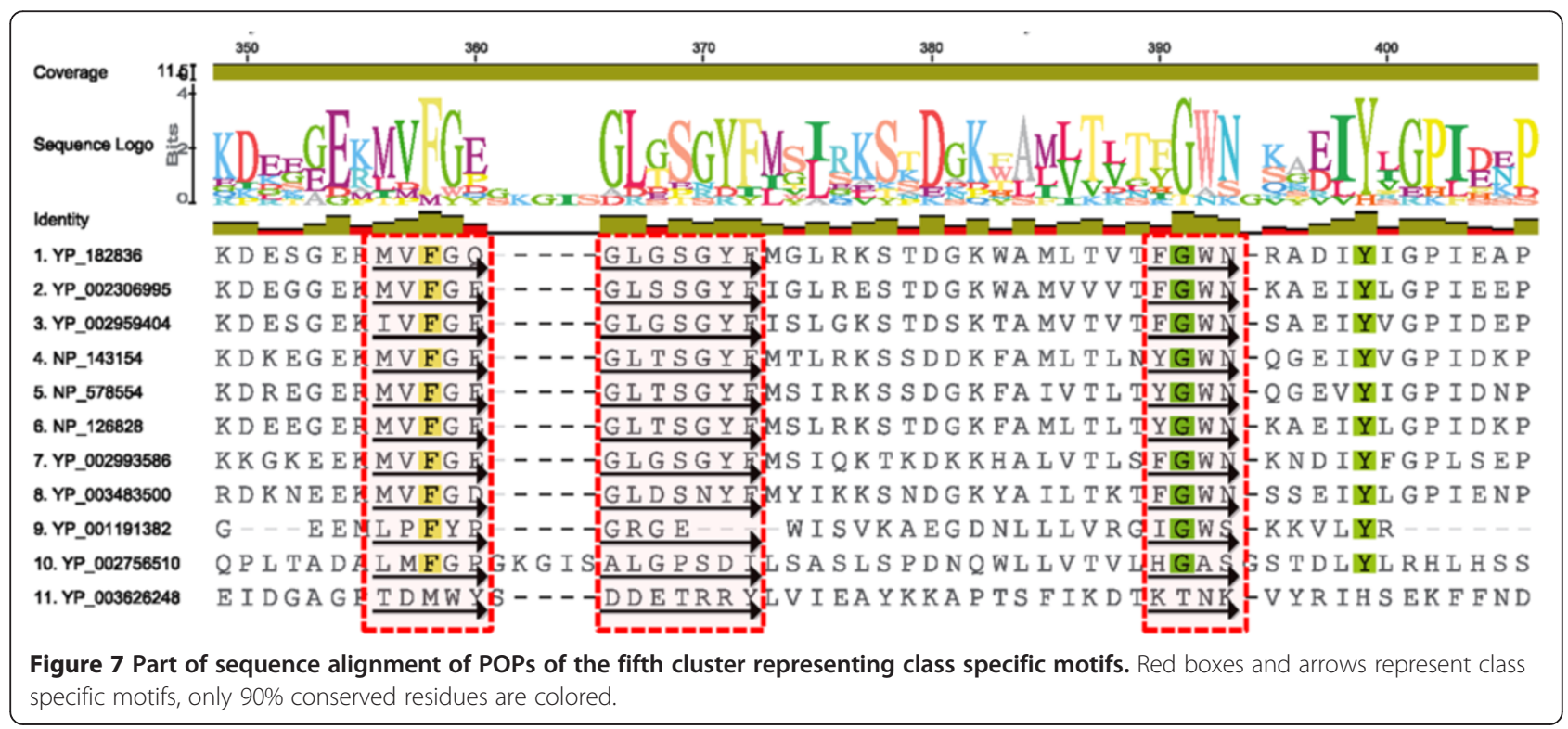




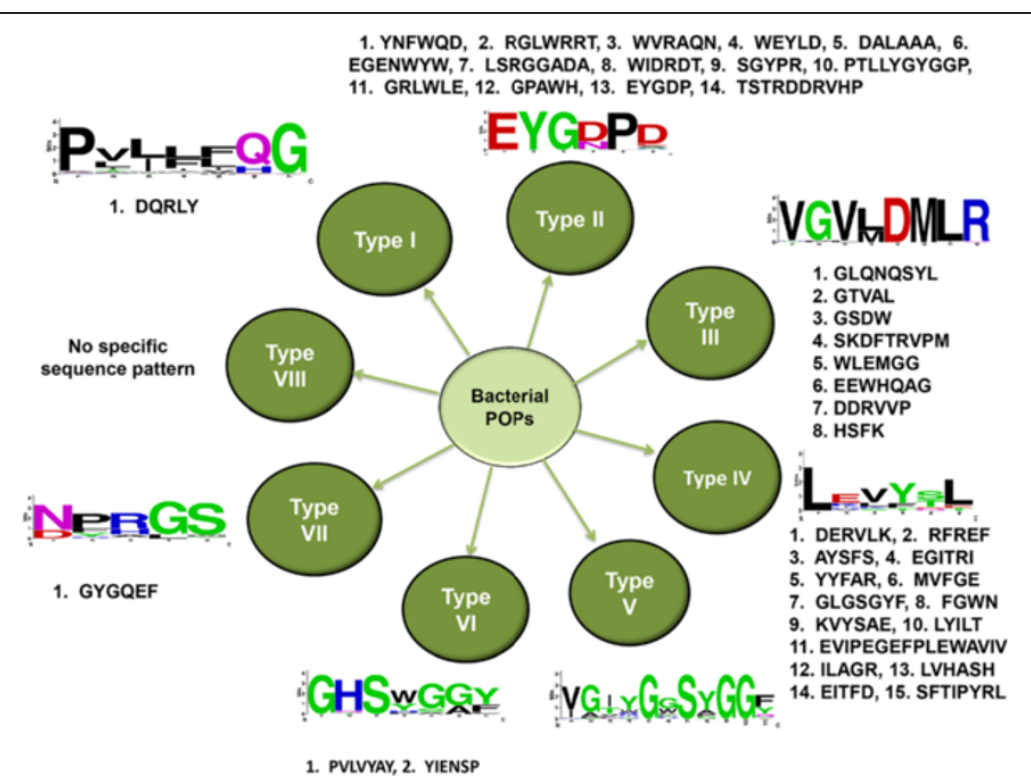

Figure 8 Different subtypes of bPOPs and the motifs associated with them. Most frequently observed motif is shown in WebLogo. Details of these motifs are present in Additional files 4, 9 and 10.

of POPs within a genome. Overall, out of 269 identified bacterial genomes with annotated POPs, 148 had a single copy of POP gene. The overrepresentation of POP was particularly observed in genus Shewanella of Gammaproteobacteria, where most of the species had multiple copies of POP gene. One of the interesting examples of multi-POP proteome was Shewanella woodyi with 16 POPs sharing an average sequence identity of $15 \%$ (ranging from 8 to 35\%). Moreover, we could identify 12 copies of POP gene in Shewanella piezotolerans, and 10 copies each in Shewanella pealeana and Shewanella sediminis. Besides genus Shewanella, 15 POP genes were also identified in Solibacter usitatus. High sequence variations in paralogs of POP suggested that they are not closely related to each other, except in S. thermophilus genome (Figure 9, Additional file 4). These multiple POPs within a genome also differ in their cellular localizations (Additional file 1-S1a).

\section{Horizontal gene transfer as a driving force for the expansion of POP gene family in bacteria}

Examination of the complete genomes of bacterial and archaeal lineages showed considerable variations in the number of annotated POP genes within a genome. Horizontal gene transfer (HGT) and gene duplication are the two driving forces, which may lead to expansion of gene families in prokaryotic systems [49]. We have studied the expansion of POP gene family in more detail using POP rich genus Shewanella. Members of genus Shewanella have been described from diverse habitats, including deep coldwater marine environments to shallow Antarctic ocean habitats, to hydrothermal vents and freshwater lakes [50].
We examined sequence similarity and chromosomal positioning to determine if HGT is prevalent in these genomes. Chromosomal mapping of 16 annotated POP genes of S. woodyi depicted non-co-localization, representing possible HGT events during evolution (Additional file 14). Only two genes (6118839 and 6118846 bearing a low sequence identity of $20 \%$ ) were found to be slightly closer on the genome, still separated by six other genes. Similar patterns were also observed in POP genes of other species of Shewanella. This suggests possibility of multiple HGT events during the evolution of these bacteria (Additional file 1-S1b and 4).

\section{Annotation of uncharacterized POP homologs of bacterial lineages}

During sensitive sequence searches, we could identify many hypothetical proteins with POP-like signatures. We have implemented various approaches such as protein domain identification, secondary structure prediction, protein fold prediction and GO annotation mapping to characterize 38 hypothetical sequences as POPs and 159 proteins as $\alpha / \beta$ hydrolases [29,51-53]. A hypothetical sequence was annotated as POP if an annotated POP query picked the sequence at least at an E-value of $10^{-3}$ and it had similar domain architecture (with both $\alpha / \beta$ hydrolase and $\beta$-propeller domains). During this analysis some partial POPs comprising of only catalytic domain were also identified (Additional file 1, S1c). RPS-BLAST (Reversed Position Specific BLAST) using four different profiles (annotated POPs, ACCs, DPPs and other hydrolases of $\alpha / \beta$ hydrolase superfamily) was carried out to further scan 


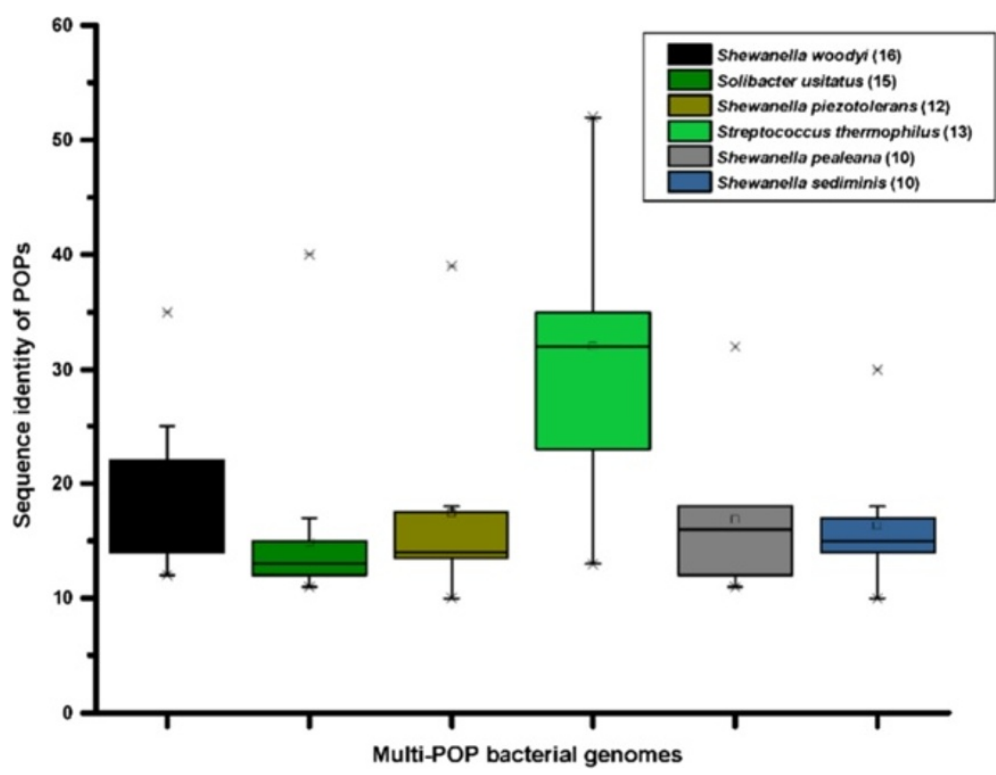

Figure 9 Sequence variations of the POPs of multi-POP bacterial genomes. Numbers of putative POPs identified in these genomes are shown in brackets.

each unannotated sequence, thereby confirming that these sequences are $\alpha / \beta$ hydrolase superfamily members [31].

\section{Limitations of the computational methods used in this study}

Although we have used multiple methods for the detailed analysis of POP homologs from the bacterial and archaeal lineages, yet the current study has certain limitations. Instead of relying on any one-sequence search method, here, we have employed multiple sequence search algorithms to detect all possible homologs. We validated all the obtained hits by mapping functional domains and active site residues, yet the possibility of obtaining false positives cannot be ignored.

The complete absence of POP genes from some of the bacterial phyla could be because of the caveats of the sequence search algorithms. It is possible that POPs of such phyla are so diverged that most of the methods failed to identify them, including current remote homology detection methods. Therefore, further experimental characterization of these genomes is essential to conclude the presence or absence of POPs. The available computational methods to predict protein domains, cellular localizations and signal peptides of protein sequences are also associated with wrong predictions.

During this study, we have also encountered many incomplete POP sequences with either missing N-terminal hydrolase domain (e.g. YP_001519174.1, Acaryochloris marina) or with incomplete propeller domain (e.g. YP_003320038.1, Sphaerobacter thermophilus) or with only hydrolase domain (e.g. Mycoplasma genomes). These partial POPs could be due to errors in the available gene prediction algorithms. Additionally, wrong or incomplete annotation of collected protein sequences could also lead to another source of error. Experimental validation of these reported sequences would help in improving the current annotations of the corresponding genomes.

\section{Conclusion}

In this study, we have performed an exhaustive computational analysis of POPs in prokaryotic lineages. Our analysis revealed wide distribution, high diversity and functional importance of POPs in the analysed bacterial and archaeal genomes. Many novel domain combinations were identified in bPOPs, emphasizing the need for systematic studies to understand them further. In addition, we noticed different selection pressures on propeller and hydrolase domains.

The POP family has primarily expanded by multiple HGT events. POP paralogs differ considerably with respect to sequence, cellular localization and domain architectures, suggesting functional divergence and maintenance by natural selection $[54,55]$. Finally, the proposed classification of bPOPs will help in understanding the sequence specific characteristics and structural differences of these POPs.

In conclusion, our systematic analysis of POPs in bacterial and archaeal species will aid in a better understanding of these proteins. This bacterial POP repertoire will also facilitate comparative and functional genomics studies, and experimental characterization of unique domain combinations. With the rapidly growing numbers of sequenced genomes, our work can be considered as a 
benchmark in the extension of such analysis to other less-studied protein families.

\section{Methods}

\section{Classification of bacterial proteomes}

1,202 prokaryotic (1,112 bacterial, 90 archaeal) proteomes were downloaded from NCBI. These proteomes were further classified according to NCBI Taxonomy database into 23 bacterial and 4 archaeal phyla. These bacterial species include diverse groups of extremophiles, pathogens, model organisms and symbiotic bacteria.

\section{Search for POP homologs in bacterial genomes}

All the prokaryotic proteomes were scanned for the presence of POPs using BLAST and HMM-SEARCH (Additional file 15) [29,31]. Instead of relying on a single method, multi-search procedures were employed to identify POP homologs from the bacterial proteomes. Sequence searches were performed at two different E-values to identify POP homologs from all the targeted proteomes. These methods include:

a) Direct approach: A direct approach was implemented by extracting the annotated POP sequences from the proteomes. If a proteome was found to have an annotated POP, it was considered as a query sequence to obtain more POP homologs. For identification of POP homologs Phmmer and Jackhmmer (iterative searching as PSI-BLAST) of the HMMER suite were used at stringent E-values of $10^{-3}$ and $10^{-10}$ [29]. Homologs were collected according to phyla and redundant sequences from each phylum were removed with CD-HIT [56]. This approach helped in identification of POP homologs where an annotated POP could already be identified.

b) Profile based approach: Bacterial and archaeal specific profiles were generated with a member (POP) from each phylum using HMMbuild. This could help in picking nearest POP homologs from all the collected bacterial proteomes. Besides archaeal and bacterial-specific profiles, an integrated profile was also generated using bacterial, archaeal and eukaryotic POPs. Furthermore, HMMsearch was performed using these three different profiles at two different $\mathrm{E}$-values of $10^{-3}$ and $10^{-10}$. This approach helped in picking homologs from proteomes, where annotated POPs were absent.

\section{Enrichment of true homologs}

Homologs collected at the relaxed E-value of $10^{-3}$ were further confirmed as 'true homologs' by constructing the database of sequences obtained at stringent E-value of $10^{-10}$. All the sequences found at $\mathrm{E}$-value of $10^{-3}$ were considered as query and BLAST was performed against the database of 'true homologs'. Domain definitions were identified using HMMScan against Pfam database for all the collected hits [57]. A hit was considered as a 'true' hit if it had both POP and $\alpha / \beta$ hydrolase domains.

To be sure that none of the POPs were missed, BLASTP was also performed against some of the selected proteomes. Furthermore, some phyla were also screened using an indirect approach of appending bacterial proteomes to PALI + database, which comprises of trusted homologs of proteins of known three-dimensional structures [31].

\section{Relative density of distribution of POP homologs in bacterial genomes}

Relative abundance of POP homologs was calculated using relative density. Relative density is defined as the total number of serine proteases (POP homologs) identified in a taxonomic lineage by the total number of genomes of that lineage, which were considered for the study. Relative occurrence is defined as the total number of POP homologs identified in a taxonomic lineage by the total number of gene products in a taxonomic lineage.

\section{Assignment of co-existing domains, transmembrane regions and signal peptides}

Domain assignments were mapped using HMMPfam (E-value of $10^{-3}$ ), which scans the sequences against HMMs of Pfam database. Transmembrane regions of all the hits were examined using TMHMM [36], which is a highly accurate HMM based method to predict transmembrane regions. Since POP is found to have protein-sorting signals, all the collected sequences were searched for the signal peptides using SignalP [58]. SignalP is the most accurate program based on neural networks to clearly distinguish signal peptides from the transmembrane regions [59]. Secondary structure prediction and fold assignments of unannotated proteins were carried out using PSIPRED and GenTHREADER, respectively [51,52].

\section{Multiple sequence alignment and phylogenetic analysis}

Multiple sequence alignment was carried out using MUSCLE [60]. The multiple sequence alignment was further utilized for performing phylogenetic analysis of collected POP homologs using MEGA5 [61]. In this analysis, we have employed neighbor-joining method, where clusters with bootstrap value greater than $50 \%$ were considered for the detailed analysis. Multiple sequence alignment was represented using WebLogo and Geneious [62]. iTOL (Interactive Tree Of Life) was used for mapping domain architecture on the species tree of bacteria and archaea [63]. MODELLER was employed for homology modeling of POP sequence, where bPOP (PDB id: 2BKL) was chosen as a template [64]. Modeled POP structures were further used for mapping sequence motifs. 
Functionally important residues were scored using Scorecons, where a score of more than 0.7 was considered to be significant [65].

\section{Additional files}

Additional file 1: Table S1a. Sequenced bacterial and archaeal genomes analysed in this study. Table S1b: BLAST searches of Shewanella genomes, cellular localization and domain architecture of multiple POP proteins of Shewanella. Table S1c Annotated hypothetical proteins.

Additional file 2: Distribution of the sequenced genomes of bacterial and archaeal lineages. Distribution of sequenced bacterial (A) and archaeal (B) genomes

Additional file 3: Schematic representation of the pipeline followed to collect true homologs.

Additional file 4: A. Enrichment of true homologs. B. Relative abundance and occurrence. $\mathbf{C}$. Other domain architectures of POP homologs. D. Different cellular localization. E. Cluster-wise sequence identity. F. Structural mapping of sequence motifs of each cluster. G Functional domains of annotated bacterial POPs are conserved and glycine rich. H. Divergence of POP family members. I. Detailed analysis of POPs of Shewanella. J. Sequence similarity searches to understand HGT events.

Additional file 5: Distribution of POP-family members in different bacterial and archaeal phyla.

Additional file 6: Domain architecture of POP homologs. Abbreviations: POP_C-prolyl oligopeptidase C-terminal, DPP_N-Dipeptidyl peptidase N-terminal, WD-WD domain, ABH-a/ß hydrolase, DLH-Dienelactone hydrolase, EstPHB-Esterase PHB, Xpro-X-Pro dipeptidyl-peptidase, ABC- $A B C$ transporter, TFB- transcription regulatory domain, TAP- TAP-like protein, TPR- Tetratrico peptide repeats, CNB- cyclicnucleotide binding, Osmc- OsmC like protein, ASST-Arylsulfo transferase, KAS- beta-ketoacyl synthase, AT- Acyl transferase, AD- Alcohol dehydrogenase, ZnD-Zinc binding dehydrogenase Branched chain aa-Branched chain amino acid, PRT- Phosphoribosyl transferase, DUF- Domain of unidentified function, BRP-Bacterial regulatory protein, FGS- Formylglycine generating sulfatase, S-layer- S-layer homology, TRD-Transcriptional regulatory domain, SMP-SMP-30/gluconolactonase/LRE-like region.

Additional file 7: Cellular localization of annotated bPOPs.

Additional file 8: Detailed phylogeny of annotated bPOPs.

Color code: Thermotogae-cyan, Firmicutes-lime, Chloroflexi-green, Deinococcus-thermus-blue, Chlorobi-magenta, Actinobacteria-blue, Acidobacteria-yellow, Alphaproteobacteria-teal, Betaproteobacteria-grey, Gammaproteobacteria-olive, Deltaproteobacteria-blue, Bacteriodetes-black, Planctomycetes-black, Cyanobacteria-purple, Gemmatimonadetes-Red branch with species name in black, Spirochaetes-pink branch with species name in black, Fibrobacteres-light grey, Archaebacteria-red.

Additional file 9: Sequence alignment and class specific motifs of each cluster. An arrow represents class specific motifs.

Additional file 10: Cluster-wise mapping of sequence motifs on the structure of POPs.

Additional file 11: A) Mapping of non-permissible amino acid replacement sites on bPOP structure. Color code: Amino acid replacement sites-red, functionally important residues-cyan and green, active site-magenta, catalytic domain-yellow and propeller domain-blue. B) Cluster-wise conservation and replacement of functionally important residues. Top row shows functionally important residues as reported in mammalian POPs. Active site residues are represented in pink. Non-permissible amino acid replacements are shown in yellow. Numbers represent percentage conservation in different clusters.

Additional file 12: Phylogenetic analysis of POP family members. Color code: POP-blue, DPP-red, ACC-magenta, OPB-green, carboxyesterase-brown.
Additional file 13: Detailed phylogeny of POP family members.

Color code: POP-blue, DPP-red, ACC-magenta, OPB-green,

carboxyesterase-brown.

Additional file 14: Chromosomal mapping of 16 POP genes of Shewanella woodyi. Color code: Purple and green color represents GC content and GC skew in this genome.

Additional file 15: Schematic of sequence searches followed in this work.

\section{Competing interests}

The authors declare that they have no competing interests.

\section{Authors' contributions}

RS and SK conceived the study. SK performed the study. RS and SK analysed the data. SK wrote the first draft of the manuscript. RS revised the final version of the text. Both authors read and approved the final manuscript.

\section{Acknowledgements}

SK acknowledges a PhD fellowship from Department of Biotechnology (DBT), India. RS and SK acknowledge financial and infrastructural support from NCBS-TIFR

\section{Author details}

'National Centre for Biological Sciences, Tata Institute of Fundamental Research, GKVK Campus, Bellary Road, Bangalore 560065, India. ${ }^{2}$ Current address: Helen Diller Family Comprehensive Cancer Center, University of California, San Francisco, CA 94158, USA.

Received: 22 July 2014 Accepted: 9 October 2014

Published: 18 November 2014

\section{References}

1. Callis J: Regulation of protein degradation. Plant Cell 1995, 7:845-857.

2. Barrett AJ, Rawlings ND, O'Brien EA: The MEROPS database as a protease information system. J Struct Biol 2001, 134:95-102.

3. Gottesman S: Proteolysis in bacterial regulatory circuits. Annu Rev Cell Dev Biol 2003, 19:565-587.

4. García-Horsman JA, Männistö PT, Venäläinen Jl: On the role of prolyl oligopeptidase in health and disease. Neuropeptides 2007, 41:1-24.

5. Hedstrom L: Serine protease mechanism and specificity. Chem Rev 2002, 102:4501-4524.

6. Jenal U, Hengge-Aronis R: Regulation by proteolysis in bacterial cells. Curr Opin Microbiol 2003, 6:163-172.

7. Hengge R, Bukau B: Proteolysis in prokaryotes: protein quality control and regulatory principles. Mol Microbiol 2003, 49:1451-1462.

8. Banbula A, Bugno M, Goldstein J, Yen J, Nelson D, Travis J, Potempa J: Emerging family of proline-specific peptidases of Porphyromonas gingivalis: purification and characterization of serine dipeptidyl peptidase, a structural and functional homologue of mammalian prolyl dipeptidyl peptidase IV. Infect Immun 2000, 68:1176-1182.

9. Caler EV, Vaena de Avalos S, Haynes PA, Andrews NW, Burleigh BA Oligopeptidase B-dependent signaling mediates host cell invasion by Trypanosoma cruzi. EMBO J 1998, 17:4975-4986.

10. Walter R, Shlank H, Glass JD, Schwartz IL, Kerenyi TD: Leucylglycinamide released from oxytocin by human uterine enzyme. Science 1971 173:827-829.

11. Rawlings ND, Polgar L, Barrett AJ: A new family of serine-type peptidases related to prolyl oligopeptidase. Biochem J 1991, 279(Pt 3):907-908

12. Camargo AC, Caldo H, Reis ML: Susceptibility of a peptide derived from bradykinin to hydrolysis by brain endo-oligopeptidases and pancreatic proteinases. J Biol Chem 1979, 254:5304-5307.

13. Venäläinen Jl, Juvonen RO, Männistö PT: Evolutionary relationships of the prolyl oligopeptidase family enzymes. Eur J Biochem FEBS 2004 271:2705-2715

14. Fülöp V, Böcskei Z, Polgár L: Prolyl oligopeptidase: an unusual beta-propeller domain regulates proteolysis. Cell 1998, 94:161-170

15. Shan L, Mathews II, Khosla C: Structural and mechanistic analysis of two prolyl endopeptidases: role of interdomain dynamics in catalysis and specificity. Proc Natl Acad Sci U S A 2005, 102:3599-3604. 
16. Li M, Chen C, Davies DR, Chiu TK: Induced-fit mechanism for prolyl endopeptidase. J Biol Chem 2010, 285:21487-21495.

17. Shan L, Marti T, Sollid LM, Gray GM, Khosla C: Comparative biochemical analysis of three bacterial prolyl endopeptidases: implications for coeliac sprue. Biochem J 2004, 383(Pt 2):311-318.

18. Cunningham DF, O'Connor B: Proline specific peptidases. Biochim Biophys Acta BBA - Protein Struct Mol Enzymol 1997, 1343:160-186.

19. Polgár L: A potential processing enzyme in prokaryotes: oligopeptidase B, a new type of serine peptidase. Proteins 1997, 28:375-379.

20. Jones WM, Scaloni A, Manning JM: Acylaminoacyl-peptidase. Methods Enzymol 1994, 244:227-231.

21. Elovson J: Biogenesis of plasma membrane glycoproteins. Purification and properties of two rat liver plasma membrane glycoproteins. J Biol Chem 1980, 255:5807-5815.

22. Durinx C, Lambeir AM, Bosmans E, Falmagne JB, Berghmans R, Haemers A, Scharpé S, De Meester I: Molecular characterization of dipeptidyl peptidase activity in serum: soluble CD26/dipeptidyl peptidase IV is responsible for the release of X-Pro dipeptides. EUr J Biochem FEBS 2000, 267:5608-5613.

23. Aertgeerts K, Ye S, Shi L, Prasad SG, Witmer D, Chi E, Sang B-C, Wijnands RA, Webb DR, Swanson RV: N-linked glycosylation of dipeptidyl peptidase IV (CD26): effects on enzyme activity, homodimer formation, and adenosine deaminase binding. Protein Sci Publ Protein Soc 2004, 13:145-154.

24. Rea D, Fülöp V: Structure-function properties of prolyl oligopeptidase family enzymes. Cell Biochem Biophys 2006, 44:349-365.

25. Wilk S: Prolyl endopeptidase. Life Sci 1983, 33:2149-2157.

26. Kaukinen K, Lindfors K, Mäki M: Advances in the treatment of coeliac disease: an immunopathogenic perspective. Nat Rev Gastroenterol Hepatol 2014, 11:36-44

27. Kumagai Y, Konishi K, Gomi T, Yagishita H, Yajima A, Yoshikawa M: Enzymatic properties of dipeptidyl aminopeptidase IV produced by the periodontal pathogen Porphyromonas gingivalis and its participation in virulence. Infect Immun 2000, 68:716-724

28. Caler EV, Morty RE, Burleigh BA, Andrews NW: Dual role of signaling pathways leading to $\mathrm{Ca} 2+$ and cyclic AMP elevation in host cell invasion by trypanosoma cruzi. Infect Immun 2000, 68:6602-6610.

29. Eddy SR: Profile hidden Markov models. Bioinforma Oxf Engl 1998, 14:755-763.

30. Henikoff JG, Henikoff S: Using substitution probabilities to improve position-specific scoring matrices. Comput Appl Biosci CABIOS 1996, 12:135-143

31. Altschul SF, Madden TL, Schäffer AA, Zhang J, Zhang Z, Miller W, Lipman DJ Gapped BLAST and PSI-BLAST: a new generation of protein database search programs. Nucleic Acids Res 1997, 25:3389-3402

32. Balaji S, Sujatha S, Kumar SSC, Srinivasan N: PALI-a database of Phylogeny and ALlgnment of homologous protein structures. Nucleic Acids Res 2001, 29:61-65.

33. Tenorio-Laranga J, Venäläinen Jl, Männistö PT, García-Horsman JA: Characterization of membrane-bound prolyl endopeptidase from brain. FEBS J 2008, 275:4415-4427.

34. O'Leary RM, Gallagher SP, O'Connor B: Purification and characterization of a novel membrane-bound form of prolyl endopeptidase from bovine brain. Int I Biochem Cell Biol 1996, 28:441-449.

35. Krogh A, Larsson B, von Heijne G, Sonnhammer EL: Predicting transmembrane protein topology with a hidden Markov model: application to complete genomes. J Mol Biol 2001, 305:567-580.

36. Murwantoko YM, Ueta Y, Murasaki A, Kanda H, Oka C, Kawaichi M: Binding of proteins to the PDZ domain regulates proteolytic activity of HtrA1 serine protease. Biochem J 2004, 381(Pt 3):895-904.

37. Spiers A, Lamb HK, Cocklin S, Wheeler KA, Budworth J, Dodds AL, Pallen MJ, Maskell DJ, Charles IG, Hawkins AR: PDZ domains facilitate binding of high temperature requirement protease $\mathrm{A}(\mathrm{HtrA})$ and tail-specific protease (Tsp) to heterologous substrates through recognition of the small stable RNA A (ssrA)-encoded peptide. J Biol Chem 2002, 277:39443-39449.

38. Blatch GL, Lässle M: The tetratricopeptide repeat: a structural motif mediating protein-protein interactions. BioEssays News Rev Mol Cell Dev Biol 1999, 21:932-939.

39. Das AK, Cohen PW, Barford D: The structure of the tetratricopeptide repeats of protein phosphatase 5: implications for TPR-mediated protein-protein interactions. EMBO J 1998, 17:1192-1199.
40. Mittl PRE, Schneider-Brachert W: Sel1-like repeat proteins in signal transduction. Cell Signal 2007, 19:20-31

41. Neer EJ, Schmidt CJ, Nambudripad R, Smith TF: The ancient regulatory-protein family of WD-repeat proteins. Nature 1994, 371:297-300.

42. Smith TF, Gaitatzes C, Saxena K, Neer EJ: The WD repeat: a common architecture for diverse functions. Trends Biochem Sci 1999, 24:181-185.

43. Janda L, Tichý P, Spizek J, Petrícek M: A deduced Thermomonospora curvata protein containing serine/threonine protein kinase and WDrepeat domains. J Bacteriol 1996, 178:1487-1489.

44. Kajava AV: Review: proteins with repeated sequence-structural prediction and modeling. J Struct Biol 2001, 134:132-144.

45. Schell MA: Molecular biology of the LysR family of transcriptional regulators. Annu Rev Microbiol 1993, 47:597-626.

46. Aravind L, Anantharaman V, Balaji S, Babu MM, lyer LM: The many faces of the helix-turn-helix domain: transcription regulation and beyond. FEMS Microbiol Rev 2005, 29:231-262.

47. Laub MT, Goulian M: Specificity in two-component signal transduction pathways. Annu Rev Genet 2007, 41:121-145.

48. Yu NY, Wagner JR, Laird MR, Melli G, Rey S, Lo R, Dao P, Sahinalp SC, Ester M, Foster LJ, Brinkman FSL: PSORTb 3.0: improved protein subcellular localization prediction with refined localization subcategories and predictive capabilities for all prokaryotes. Bioinformatics 2010, 26:1608-1615.

49. Treangen TJ, Rocha EPC: Horizontal transfer, not duplication, drives the expansion of protein families in prokaryotes. PLoS Genet 2011, 7:e1001284.

50. Dikow RB: Genome-level homology and phylogeny of Shewanella (Gammaproteobacteria: Iteromonadales: Shewanellaceae). BMC Genomics 2011, 12:237.

51. McGuffin LJ, Bryson K, Jones DT: The PSIPRED protein structure prediction server. Bioinforma Oxf Engl 2000, 16:404-405.

52. Jones DT: GenTHREADER: an efficient and reliable protein fold recognition method for genomic sequences. J Mol Biol 1999, 287:797-815.

53. Carbon S, Ireland A, Mungall CJ, Shu S, Marshall B, Lewis S: AmiGO: online access to ontology and annotation data. Bioinformatics 2009, 25:288-289.

54. Näsvall J, Sun L, Roth JR, Andersson DI: Real-time evolution of new genes by innovation, amplification, and divergence. Science 2012, 338:384-387.

55. Collins RE, Merz H, Higgs PG: Origin and evolution of gene families in Bacteria and Archaea. BMC Bioinformatics 2011, 12(Suppl 9):S14.

56. Li W, Godzik A: Cd-hit: a fast program for clustering and comparing large sets of protein or nucleotide sequences. Bioinforma Oxf Engl 2006, 22:1658-1659.

57. Finn RD, Mistry J, Tate J, Coggill P, Heger A, Pollington JE, Gavin OL, Gunasekaran P, Ceric G, Forslund K, Holm L, Sonnhammer ELL, Eddy SR, Bateman A: The Pfam protein families database. Nucleic Acids Res 2009, 38(Database):D211-D222

58. Petersen TN, Brunak S, von Heijne G, Nielsen H: SignalP 4.0: discriminating signal peptides from transmembrane regions. Nat Methods 2011, 8:785-786

59. Nielsen $\mathrm{H}$, Engelbrecht J, Brunak S, von Heijne G: Identification of prokaryotic and eukaryotic signal peptides and prediction of their cleavage sites. Protein Eng 1997, 10:1-6.

60. Edgar RC: MUSCLE: multiple sequence alignment with high accuracy and high throughput. Nucleic Acids Res 2004, 32:1792-1797.

61. Tamura K, Peterson D, Peterson N, Stecher G, Nei M, Kumar S: MEGA5: molecular evolutionary genetics analysis using maximum likelihood, evolutionary distance, and maximum parsimony methods. Mol Biol Evol 2011, 28:2731-2739.

62. Crooks GE, Hon G, Chandonia J-M, Brenner SE: WebLogo: a sequence logo generator. Genome Res 2004, 14:1188-1190.

63. Letunic I, Bork P: Interactive Tree Of Life (iTOL): an online tool for phylogenetic tree display and annotation. Bioinformatics 2007, 23:127-128.

64. Sali A, Blundell TL: Comparative protein modelling by satisfaction of spatial restraints. J Mol Biol 1993, 234:779-815.

65. Valdar WSJ: Scoring residue conservation. Proteins 2002, 48:227-241.

doi:10.1186/1471-2164-15-985

Cite this article as: Kaushik and Sowdhamini: Distribution, classification domain architectures and evolution of prolyl oligopeptidases in prokaryotic lineages. BMC Genomics 2014 15:985. 